\section{ACUTE INFLAMMATORY INJURY OF IMMATURE CEREBRAL WHITE MATTER ASSESSED BY SPECTROSCOPY AT 9.4T}

G. Lodygensky' ${ }^{1}$, N. Kunz ${ }^{2}$, S. Sizonenko ${ }^{3}$, R. Gruetter ${ }^{2}$, P.S. Hüppi ${ }^{3}$

${ }^{1}$ Pediatrics, NICU, University of Geneva, Geneva, ${ }^{2}$ LIFMET, CIBM, Ecole Polytechnique de Lausanne, Lausanne, ${ }^{3}$ Pediatris, Division of Development and Growth, University of Geneva, Geneva, Switzerland

Intracallosal injection of lipopolysaccharide (LPS) is a well-established model of white matter injury in the developing brain. Early alterations of tissue metabolism after an inflammatory insult are unknown. As such, we sought to define its in vivo spectroscopic signature 24 hours after injection. 8 Wistar rats injected under ultrasound guidance with $1 \mathrm{mg} / \mathrm{kg}$ of LPS were compared to 8 rats injected with normal saline (CTR) on day of life 3 . Both groups were scanned on day of life 4 at 9.4 Tesla with a short echo-time $(2.75 \mathrm{~ms})$ spin-echo, full intensity acquired localized pulse sequence (SPECIAL) that allowed the acquisition of a 7.5 microliter voxel covering the corpus callosum. Metabolite concentrations were quantified using the LCModel. A Cramér-Rao lower bounds $<20$ identified quantifiable metabolites. Preliminary spectroscopy results, 24 hours after LPS injection, identified 19 peaks with high accuracy in the corpus callosum. Among those metabolites, acute LPS exposure caused an increase in lactate and macromolecules. This increase reached significance with the lactate quantification (Lac LPS $3.48 \pm 0.52 \mu \mathrm{mol} / \mathrm{g} \pm \mathrm{SE}$, Lac CTR $1.89 \pm 0.25 \mu \mathrm{mol} / \mathrm{g} \pm \mathrm{SE}, p 0.01$ ) but was only a trend for quantification of macromolecules (20\% relative increase, Mac LPS $1.8 \pm 0.1 \mu \mathrm{mol} / \mathrm{g}$ \pm SE, Mac CTR $1.51 \pm 0.11 \mu \mathrm{mol} / \mathrm{g} \pm$ SE, $p$ 0.08). A higher number of animals will be scanned to estimate the effect of gender in relation to injury. Ultimately this spectroscopic signature could be used to estimate the severity of white matter injury in preterm infants.

\section{BRAIN PLASTICITY AFTER PRETERM BIRTH: A FMRI STUDY OF THE VISUAL PATHWAY}

R. Ha-Vinh Leuchter ${ }^{1,2}$, T. Chaminade ${ }^{3}$, V. Millet ${ }^{1}$, C. Deruelle ${ }^{3}$

${ }^{1}$ Médecine Néonatale, Assistance PubliqueHôpitaux de Marseille, Marseille, France, ${ }^{2}$ Hôpital des Enfants, HUG, Geneva, Switzerland, ${ }^{3}$ Institut de Neurosciences Cognitives de la Méditeranée, Marseille, France

Objective: Although a large number of very preterm children follow a normal school cursus, they nevertheless present cognitive difficulties affecting visuo-spatial abilities especially. In this study, we investigated whether these visuo-spatial difficulties could originate in a dysfunction of the visual cerebral pathways (i.e., dorsal and ventral pathways).

Methods: A group of 7 young adults (MA $=19.4 \pm$ 0.8 years) who were born preterm (GA: $29.9 \pm 1.7$ wks), and a group of 7 term born controls (MA = $20.22 \pm 1.5$ years ; GA: $39.14 \pm 1.4$ wks) underwent a complete neurologic examination, an IQ battery, a standardized motor test and a task which was developed to investigate specific cerebral responses of the dorsal and ventral visual stream, both in terms of processing of visual information (bottom-up) and allocation of attention (top-down).

Results: No significant difference between groups was found for IQ, motor performances and behavioral dorsal and ventral functionning. The fMRI data showed an additional activation of ventral occipital areas during dorsal stream tasks in preterms compared to their controls.

Conclusion: By showing an additional ventral activation in a task that typically relies on dorsal stream activation, our finding strongly suggest that preterm adults use alternative strategies to behaviorally perform as term born adults. They also suggest that the dorsal stream is more vulnerable during early development and that ventral stream brain areas are more susceptible to plasticity than their dorsal counterparts. 